\title{
Peripheral arterial disease and osteoporosis in older adults: the Rancho Bernardo Study
}

\author{
D. von Mühlen • M. Allison • S. K. Jassal • \\ E. Barrett-Connor
}

Received: 17 November 2008 / Accepted: 4 March 2009 / Published online: 24 March 2009

(C) The Author(s) 2009. This article is published with open access at Springerlink.com

\begin{abstract}
Summary We examined the association between peripheral arterial disease (PAD) and bone health in 1,332 adults. We found a weak association between PAD and osteoporosis and bone loss only in women, but the association was not independent of age. PAD was not associated with fractures in this community-based population.

Introduction Increased rates of osteoporosis have been reported in patients with cardiovascular disease, suggesting a link between osteoporosis and atherosclerosis.

Methods We examined the association between PAD and bone health in 1,332 adults who attended a research visit in 1992-1996, when the ankle-brachial index (ABI), bone mineral density (BMD), and spine X-rays were obtained. A total of 837 participants attended a follow-up visit in 19972000 .
\end{abstract}

D. von Mühlen $(\varangle) \cdot$ E. Barrett-Connor

Division of Epidemiology, Department of Family and Preventive

Medicine, University of California,

La Jolla, San Diego, CA, USA

e-mail: dvonmuhlen@ucsd.edu

E. Barrett-Connor

e-mail: ebarrettconnor@ucsd.edu

M. Allison

Division of Preventive Medicine, Department of Family and

Preventive Medicine, University of California,

La Jolla, San Diego, CA, USA

e-mail: mallison@ucsd.edu

S. K. Jassal

Department of Medicine, University of California,

La Jolla, San Diego, CA, USA

e-mail: sjassal@ucsd.edu

S. K. Jassal

VA San Diego Healthcare System,

La Jolla, San Diego, CA, USA
Results $\mathrm{PAD}$ defined by an $\mathrm{ABI} \leq 0.90$ was present in $15.4 \%$ of the women and $13.3 \%$ of the men. Prevalence of osteoporosis was significantly higher in women with PAD compared to women without PAD $(p<0.05)$. During an average 4-year follow-up, women with PAD had a significantly higher rate of bone loss than women without $\operatorname{PAD}(p=0.05)$. The associations were no longer significant after age adjustment. In men, PAD was not associated with osteoporosis, but men with PAD had lower BMD at the femoral neck than men without PAD $(p=0.03)$. PAD was not associated with osteoporotic fractures in either sex.

Conclusion We found a weak and age-dependent association between PAD and osteoporosis in women but not men. PAD was not associated with fractures in this community-based population.

Keywords Osteoporosis · Peripheral artery disease

\section{Introduction}

Increased rates of bone loss, osteoporosis, and osteoporotic fractures have been reported in adults with cardiovascular disease, suggesting an association between osteoporosis and atherosclerosis [1-3]. A few studies have suggested an association between osteoporosis and peripheral arterial disease (PAD) in women [4-6], but studies in men yielded inconsistent results $[5,7]$. Low bone mineral content at menopause appears to be a risk factor for increased cardiovascular disease mortality in later life [8-10]. To our knowledge, the association of PAD with osteoporotic fractures has not been reported. We report here a study examining the association between PAD based on the ankle-brachial index (ABI), with measures of bone health assessed by dual energy X-ray absorptiometry (DXA) and 
fracture status in a large population-based sample of older men and women. The aims of this study were: (1) to determine whether PAD defined by several ABI cut points is associated with low bone mineral density (BMD), bone loss, and/or osteoporotic fractures; (2) to determine whether PAD can predict BMD loss or incident osteoporotic fractures; and (3) to determine whether any associations are independent of age, body mass index (BMI), and other covariates including smoking, physical activity, alcohol intake, medication use, lipid levels, blood pressure, renal function, and diabetes status.

\section{Methods}

The Rancho Bernardo Study, a cohort of Caucasian, middle to upper-middle class, community-dwelling adults in Southern California, was established in 1972; details of the initial study have been published [11]. Between 1992 and 1996 , approximately $80 \%(n=1,778)$ of surviving local residents participated in a research clinic visit. A total of 527 men and 805 women, aged 30 to 97 (mean age $=73.8$, $\mathrm{SD}=9.2$ ) completed standardized questionnaires about medical history, including osteoporotic fractures and were examined for ABI and BMD. Seventy-seven percent $(n=$ 1,096) of surviving participants returned for a follow-up visit in 1997-2000. Of these, 322 men and 515 women had BMD measurement repeated and were queried about interim OP fractures. Main reasons for nonparticipation among survivors included moving away, being too sick or too busy, or being institutionalized.

Data on the following variables were collected at baseline: age, height, weight, alcohol intake (drink alcohol three or more times/week), smoking status (current vs. not current), medications, physical activity (exercise three or more times/week), history of bone fractures and diabetes, fasting lipid levels, renal function, intermittent claudication (a symptom of severe PAD) based on the Rose questionnaire [12], BMD, and ABI (see below). Radiographs of the thoracic and lumbar spine were obtained and read by a single skeletal radiologist. Serum creatinine levels were measured by Smith Kline Beecham clinical laboratories. Creatinine clearance was calculated by the modified Cockcroft-Gault formula: $[140-$ age (in years) $] \times$ weight (in kilograms) / [72 $\times$ serum creatinine $(\mathrm{mg} / \mathrm{dl})]$ and corrected for body surface area. For women, the product was multiplied by 0.85 (a correction factor recommended for females) [13].

BMD was measured at the hip and lumbar spine using DXA (Hologic QDR model 1000; Hologic Inc., Bedford, MA, USA). Total hip BMD included the greater trochanter, femoral neck, and intertrochanter area. Bone densitometers were calibrated daily and measurements maintained within the manufacturers' precision standards. The BMD $T$ scores were expressed in standard deviations using the peak bone mass from the manufacturer's reference population. Osteoporosis was defined as BMD at the femoral neck or the hip $\geq 2.5$ standard deviations (SD) below the young adult mean.

Incident fractures and repeated BMD were determined at a follow-up visit an average of 4 years (range 1-7) later. Bone change was calculated as BMD percent change per year. Non-vehicular accident fractures occurring after age 45 were classified as osteoporotic. Ninety-five percent of self-reported fractures were confirmed by radiology reports.

\section{ABI measurements}

The ABI is a simple noninvasive method to assess the presence and extent of atherosclerosis in the lower leg. Participants were rested in the supine position for $5 \mathrm{~min}$, after which a specially trained and certified nurse used a handheld Doppler flowmeter attached to a mercury sphygmomanometer cuff to measure the blood pressure in each arm (brachial artery) and leg (posterior tibial artery). Systolic blood pressure was recorded once for each arm and twice for each leg. The ABI was calculated for each leg by dividing the higher systolic pressure of the leg by the systolic blood pressure in the arm. The lower of these two ABIs was used to define participants with PAD. The sensitivity and specificity of an $\mathrm{ABI}>0.9$ for $\mathrm{PAD}$ are $80 \%$ and $95 \%$, respectively [14]. One man and one woman had an $\mathrm{ABI}>1.3$, consistent with noncompressible arteries and were excluded from the analyses.

\section{Statistical analyses}

Descriptive analyses are expressed as mean (SD) or percentages and were compared using the Student $t$ test or chi-square tests as appropriate. Analysis of covariance was used to calculate sex- and site-specific mean BMD levels and mean annual percent change in BMD stratified by PAD status (defined as $\mathrm{ABI}>0.9$ vs. $\mathrm{ABI} \leq 0.9$ and using literature suggested cut-points of $<0.90,0.90-1.00,1.01-$ 1.10 , and $>1.10$ ) [15]. Risk factors previously shown to be associated with BMD in this cohort (age, BMI, use of calcium supplements (yes/no), exercise ( $\geq 3 /$ week), renal function, and hormone therapy use (current vs. not) as well as classic risk factors for atherosclerosis and PAD (smoking, hypertension, systolic blood pressure, TC/HDL ratio, and diabetes) were examined in separate and multivariate models. Adjustments for other possible confounders including use of thiazides, vitamin D supplements, and lipidlowering medication did not change any of the results and were not included in the final models. Adjusted multiple 


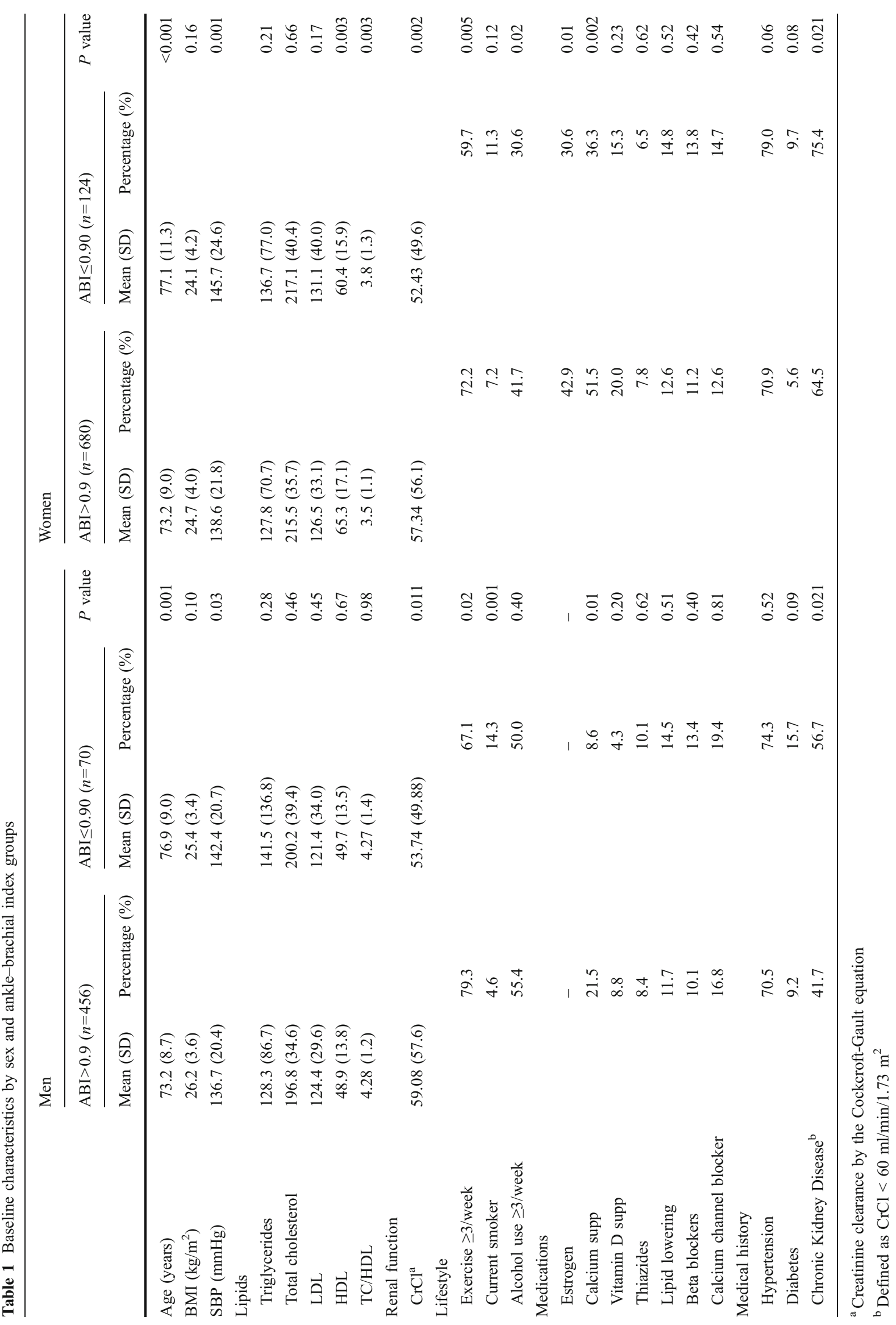




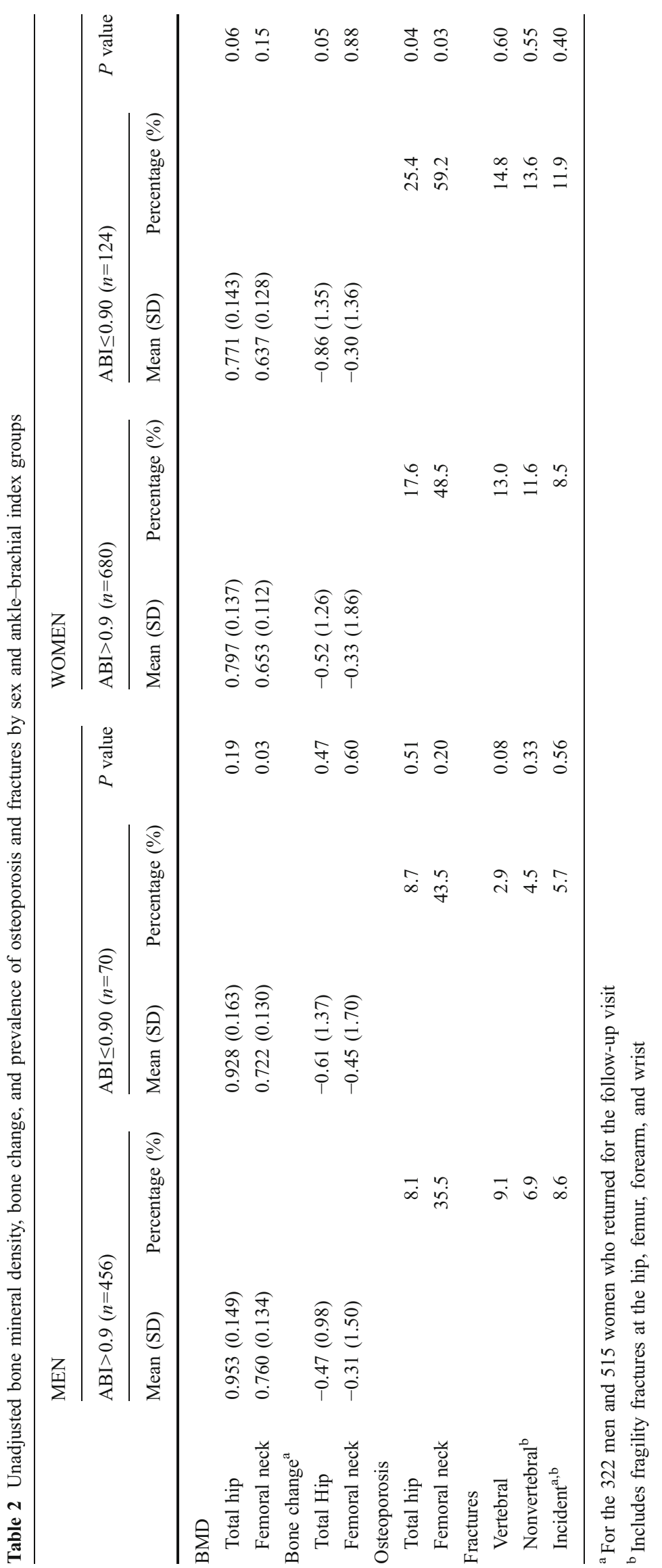


logistic regression was used to assess the contribution of PAD status to the prevalence and incidence of osteoporotic fractures. Because there were important differences in the prevalence of osteoporosis, bone loss, and PAD between men and women, all analyses were presented stratified by sex. All statistical tests were two-tailed, with statistical significance defined as $p<0.05$. SPSS (SPSS Inc., SPSS Base 15 for Windows User's Guide) and SAS (SAS Institute SAS User's Guide, Version 8.2) were used for analysis.

\section{Results}

The mean age was 74 years ( $\mathrm{SD}=9$, range 30 to 97 ). At baseline, PAD defined by an $\mathrm{ABI} \leq 0.90$ was present in $15.4 \%$ of women and $13.3 \%$ of men. No participants reported intermittent claudication. Table 1 shows that, compared to those without PAD, men and women with PAD were older $(p<0.001)$, more likely to have higher SBP $(p \leq 0.03)$ and lower levels of creatinine clearance $(p \leq 0.01)$, more likely to be sedentary $(p \leq 0.02)$, less likely to report calcium supplementation $(p<0.02)$, and more likely to have chronic kidney disease defined as $\mathrm{CrCl}<60 \mathrm{ml} / \mathrm{min} / 1.73 \mathrm{~m}^{2}$ $(p=0.02)$. Additionally, women with PAD were less likely to be current users of estrogen therapy $(p=0.01)$, had a higher TC/HDL ratio $(p=0.003)$, and were less likely to report alcohol intake $(p=0.02)$ than women without PAD. Men (but not women) with PAD were more likely to be current smokers $(p=0.001)$ than men without PAD.

Participants who did not return for the follow-up visit were older ( 75.8 vs. 72.6 years, $p<0.01$ ), had lower mean ABI (1.02 vs. $1.06, p<0.01)$ and were more likely to have categorically defined PAD $(19.5 \% 1$ vs. $11.7 \% p<0.001)$ when compared to participants who returned for the followup visit. They were also more likely to have total hip and femoral neck osteoporosis ( $18.4 \%$ vs. $12.2 \%, p=0.002$ and
$49.5 \%$ vs. $42.1 \%, p=0.03$, respectively) but had similar prevalence of vertebral and nonvertebral osteoporotic fractures.

The BMD, BMD change, and prevalent and incident osteoporotic fractures are shown in Table 2. The only statistically significant differences were that men with PAD had lower BMD at the femoral neck $(p=0.03)$, and women with PAD had a significantly higher rate of bone loss at the hip $(-0.86 \% /$ year vs. $-0.52 \% /$ year, $p=0.05)$ when compared to men and women without PAD. Compared to women without PAD, the prevalence of osteoporosis by WHO ( $T$ score) criteria at the femoral neck and hip was significantly higher in women with PAD $(59.2 \%$ vs. $48.5 \%$ and $25.4 \%$ vs. $17.6 \%$, respectively, $p<0.05$ ). There were no differences in categorically defined osteoporosis prevalence by PAD status in men. All significant associations between PAD and bone were no longer significant after adjusting for age. Further adjustments for BMI, exercise, smoking status, cholesterol/HDL ratio, hypertension, creatinine clearance, and diabetes did not materially change any of the results. Stratifying ABI by quartiles or using three categories (tertiles or $\mathrm{ABI}<0.9,0.9-1.1$, and $>1.1$ ) did not change the significance of the associations (results not shown).

At baseline, 143 participants had reported at least one clinical vertebral fracture and 126 reported a nonvertebral fracture. Incident nonvertebral fractures were reported by 70 participants. More women than men had a vertebral and/ or nonvertebral osteoporotic fracture at baseline $(13 \%$ vs. $8 \%$ and $12 \%$ vs. $7 \%$, respectively; all $p<0.01$ ), but there were no sex difference in the incidence of nonvertebral OP fractures $(8.2 \%$ in men vs. $9.0 \%$ in women, $p=0.72)$. Logistic regression models (Table 3 ) show that PAD was not associated with prevalent or incident $\mathrm{OP}$ fractures in men or women. After a mean follow-up of 4 years $(\mathrm{SD}=$ $0.9)$, BMD was the only independent variable associated with osteoporotic fractures for both sexes with higher BMD
Table 3 Odds ratio for predictors of osteoporotic fractures in men and women

Rancho Bernardo Study 19921996 and 1999-2002. Multivariable models also included current smoking, lack of exercise, hypertension, diabetes, TC/HDL, and kidney disease - all variables were not significant predictors of fractures

${ }^{*} p<0.05, * * p \leq 0.01$

\begin{tabular}{llll}
\hline & Nonvertebral fractures & Vertebral fractures & Incident nonvertebral fractures \\
\hline Men & $(n=34)$ & $(n=42)$ & $(n=26)$ \\
ABI $<0.9$ & $1.25(0.36-4.37)$ & $3.33(0.74-14.9)$ & $1.52(0.30-7.45)$ \\
Age (years) & $0.97(0.92-1.02)$ & $1.01(0.97-1.07)$ & $1.01(0.94-1.07)$ \\
BMI & $1.01(0.89-1.15)$ & $1.01(0.88-1.13)$ & $1.16(1.00-1.35)$ \\
Hip BMD & $0.18(0.01-3.20)$ & $0.03(0.002-0.49)^{* *}$ & $0.004(0.00-0.20)^{* *}$ \\
Women & $(n=92)$ & $(n=101)$ & $(n=44)$ \\
ABI $<0.9$ & $0.87(0.47-1.63)$ & $1.47(0.75-2.87)$ & $0.84(0.31-2.26)$ \\
Age (years $)$ & $1.00(0.97-1.04)$ & $1.06(1.02-1.10)^{* *}$ & $0.98(0.93-1.03)$ \\
BMI & $0.99(0.92-1.07)$ & $1.13(1.05-1.21)^{*}$ & $1.05(0.95-1.15)$ \\
Hip BMD & $0.07(0.01-0.58)^{* *}$ & $0.005(0.01-0.04)^{* *}$ & $0.12(0.01-2.30)$ \\
Current estrogen & $1.19(0.70-2.03)$ & $1.62(0.92-2.86)$ & $1.05(0.49-2.22)$ \\
\hline
\end{tabular}


associated with fewer prevalent nonvertebral and vertebral fractures in women and prevalent vertebral fractures and incident nonvertebral fractures in men. In women, age and BMI were also associated with clinical vertebral fractures.

\section{Discussion}

In this study, PAD defined as an $\mathrm{ABI} \leq 0.9$ was not independently associated with $\mathrm{BMD}$, osteoporosis, or osteoporotic fractures in either sex. In accord with other studies, hip BMD was an independent risk factor for vertebral and nonvertebral fractures in both sexes [16-20]. The increasing odds for a vertebral fracture with increasing BMI observed in women in this study were unexpected and could be spurious. A high BMI has been shown to protect the bone, and low BMI is a risk factor for osteoporotic fractures in weight-bearing appendicular bones [21, 22], but the effect of BMI on the spine has been less consistent. Three large population-based studies found a weak [23] or absent association [24, 25] between bodyweight and prevalent or incident vertebral fracture in both sexes. In contrast, increasing bodyweight was associated with a reduced risk of a first vertebral fracture in women in the Study of Osteoporotic Fractures [26]. We were unable to examine incident vertebral fractures because X-rays were not obtained in the follow-up visit.

Previous studies examining the cross-sectional association between osteoporosis and PAD have reported weak or absent associations. Vogt and collaborators [27] studied 1,292 women from the Study of Osteoporotic Fractures with a mean age of 71 years and found an association between the ABI and BMD at the femoral neck, but the association was not independent of BMI. Van der Klift and collaborators [5] studied 3,053 women and 2,215 men aged 60 to 70 years from the Rotterdam Study and found that PAD was associated with lower BMD at the femoral neck in women but not in men, with no associations found between PAD and lumbar spine in either sex. Mangiafico and collaborators [4] reported an $18.2 \%$ prevalence of PAD in women with osteoporosis versus $3.8 \%$ in women with normal BMD; lower BMD at the femoral neck was associated with PAD independent of BMI, smoking, lipid levels, blood pressure, or other risk factors for atherosclerosis.

Different results have been reported from recent small case-control studies of patients with advanced arterial disease. Pennisi and collaborators [28] studied 36 women with advanced atherosclerosis of the carotid and/or femoral artery and 30 age-matched controls and reported that carotid or femoral plaque measured by ultrasonography was associated with both regional and total body BMD. Laroche and collaborators [7] studied 18 men with symptomatic arterial disease of the lower limbs and found a decrease in bone mineral content in the more affected leg compared with the less affected leg. Ischemia was postulated to be the cause of local bone loss in these men with asymmetric PAD. Fahrleitner-Pammer and collaborators [29] examined 95 men and women with angiographically confirmed PAD and 44 controls and found that PAD was associated with lower BMD and increased bone resorption independent of BMI and other known confounders. In our study, the associations between PAD and BMD were weak and age-dependent. It is likely that people with mild or asymptomatic arterial disease do not have sufficient compromised circulation to impair bone health, unlike those in the studies above.

Overall, these data suggest that severe atherosclerosis that compromises blood flow to the lower limb may cause bone loss but that mild usually subclinical PAD does not. In a recent prospective study of 963 postmenopausal women, Tanko and collaborators [30] reported that severity of atherosclerosis in the aorta was inversely associated with BMD at the hip but not at the radius or spine and concluded that the association of aortic calcification with BMD is sitespecific. The authors speculated that aortic calcification may influence blood flow to the distal regions affecting blood supply to the hip [31]. In a large prospective study of 3,998 Chinese men and women aged 65 to 92 years, Wong and collaborators [32] reported an association between PAD and BMD at the hip, but, as in our study, this association was not independent of age, sex, bodyweight, and other risk factors. There is evidence that arterial calcification is a strong predictor of low bone mass and fragility fractures, but to our knowledge, no study has examined the association of PAD with prevalent and incident osteoporotic fractures. Patients with PAD may experience difficulties with mobility and proprioception increasing their likelihood of falls and fractures. Although we found no association between PAD and prevalent or incident osteoporotic fractures, there were relatively few fractures limiting our power.

Our study has other limitations. The Rancho Bernardo Study population is almost entirely Caucasian and middle to upper-middle class; results might not generalize to other populations. However, the prevalence of PAD was $15 \%$ in women and $13 \%$ in men-similar to PAD prevalence reported by other comparable studies [5]. Participants' mean age at baseline was 74 years, and participants who did not return for the follow-up visit were older and more likely to have PAD and osteoporotic fractures. PAD was not assessed by angiography, but others have shown a high validation of ABI with angiographic studies [33]. Finally, the mean follow-up for bone loss and incident fractures was only 4 years, and the incidence of OP fractures was low. A longer follow-up may be needed to better assess the role of PAD in the incidence of OP fractures. 
In conclusion, in these relatively healthy older adults, associations were weak and entirely explained by age. Longer, larger prospective studies are needed to determine whether asymptomatic $\mathrm{ABI}$ independently predicts bone loss and fractures in older adults. Given the increasing age in the USA, it is important to examine the association between these two chronic conditions and potential common underlying pathophysiologic mechanisms.

Acknowledgments The Rancho Bernardo Study was funded by the National Institute of Diabetes and Digestive and Kidney Diseases, grant DK31801, and the National Institute on Aging, grant AG07181. This study was partially supported by an unrestricted grant by the Alliance for Better Bone Health: Procter \& Gamble Pharmaceuticals and Sanofi-Aventis Pharmaceuticals.

\section{Conflicts of interest None.}

Open Access This article is distributed under the terms of the Creative Commons Attribution Noncommercial License which permits any noncommercial use, distribution, and reproduction in any medium, provided the original author(s) and source are credited.

\section{References}

1. Farhat GN, Strotmeyer ES, Newman AB, Sutton-Tyrrell K, Bauer DC, Harris T (2006) Volumetric and areal bone mineral density measures are associated with cardiovascular disease in older men and women: the health, aging, and body composition study. Calcif Tissue Int 79:102-111

2. Barengolts EI, Berman M, Kukreja SC, Kouznetsova T, Lin C, Chomka EV (1998) Osteoporosis and coronary atherosclerosis in asymptomatic postmenopausal women. Calcif Tissue Int 62:209-213

3. Banks LM, Lees B, MacSweeney JE, Stevenson JC (1994) Effect of degenerative spinal and aortic calcification on bone density measurements in post-menopausal women: links between osteoporosis and cardiovascular disease? Eur J Clin Invest 24:813-817

4. Mangiafico RA, Russo E, Riccobene S, Pennisi P, Mangiafico M, D'Amico F (2006) Increased prevalence of peripheral arterial disease in osteoporotic postmenopausal women. J Bone Miner Metab 24:125-131

5. van der Klift M, Pols HA, Hak AE, Witteman JC, Hofman A, de Laet CE (2002) Bone mineral density and the risk of peripheral arterial disease: the Rotterdam Study. Calcif Tissue Int 70:443-449

6. Gupta G, Aronow WS (2006) Atherosclerotic vascular disease may be associated with osteoporosis or osteopenia in postmenopausal women: a preliminary study. Arch Gerontol Geriatr 43:285-288

7. Laroche M, Pouilles JM, Ribot C, Bendayan P, Bernard J, Boccalon H (1994) Comparison of the bone mineral content of the lower limbs in men with ischaemic atherosclerotic disease. Clin Rheumatol 13:611-614

8. Browner WS, Seeley DG, Vogt TM, Cummings SR (1991) Nontrauma mortality in elderly women with low bone mineral density. Study of Osteoporotic Fractures Research Group. Lancet 338:355358
9. Kado DM, Browner WS, Blackwell T, Gore R, Cummings SR (2000) Rate of bone loss is associated with mortality in older women: a prospective study. J Bone Miner Res 15:1974-1980

10. Mussolino ME, Madans JH, Gillum RF (2003) Bone mineral density and mortality in women and men: the NHANES I epidemiologic follow-up study. Ann Epidemiol 13:692-697

11. Criqui MH, Barrett-Connor E, Austin M (1978) Differences between respondents and non-respondents in a population-based cardiovascular disease study. Am J Epidemiol 108:367-372

12. Rose G, McCartney P, Reid DD (1977) Self-administration of a questionnaire on chest pain and intermittent claudication. Br J Prev Soc Med 31:42-48

13. Hanley DA, Brown JP, Tenenhouse A, Olszynski WP, Ioannidis G, Berger C (2003) Associations among disease conditions, bone mineral density, and prevalent vertebral deformities in men and women 50 years of age and older: cross-sectional results from the Canadian Multicentre Osteoporosis Study. J Bone Miner Res 18:784-790

14. Feigelson HS, Criqui MH, Fronek A, Langer RD, Molgaard CA (1994) Screening for peripheral arterial disease: the sensitivity, specificity, and predictive value of noninvasive tests in a defined population. Am J Epidemiol 140:526-534

15. Allison MA, Laughlin GA, Barrett-Connor E, Langer R (2006) Association between the ankle-brachial index and future coronary calcium (the Rancho Bernardo study). Am J Cardiol 97:181-186

16. Leslie WD, Tsang JF, Lix LM (2008) The effect of total hip bone area on osteoporosis diagnosis and fractures. J Bone Miner Res 23 (9):1468-1476

17. Bauer DC, Gluer CC, Cauley JA, Vogt TM, Ensrud KE, Genant HK (1997) Broadband ultrasound attenuation predicts fractures strongly and independently of densitometry in older women. A prospective study. Study of Osteoporotic Fractures Research Group. Arch Intern Med 157:629-634

18. Mackey DC, Eby JG, Harris F, Taaffe DR, Cauley JA, Tylavsky FA (2007) Prediction of clinical non-spine fractures in older black and white men and women with volumetric BMD of the spine and areal BMD of the hip: the Health, Aging, and Body Composition Study*. J Bone Miner Res 22:1862-1868

19. Faulkner KG, Wacker WK, Barden HS, Simonelli C, Burke PK, Ragi S (2006) Femur strength index predicts hip fracture independent of bone density and hip axis length. Osteoporos Int 17:593-599

20. Szulc P, Munoz F, Duboeuf F, Marchand F, Delmas PD (2005) Bone mineral density predicts osteoporotic fractures in elderly men: the MINOS study. Osteoporos Int 16:1184-1192

21. Morin S, Tsang JF, Leslie WD (2008) Weight and body mass index predict bone mineral density and fractures in women aged 40 to 59 years. Osteoporos Int 20(3):363-370

22. De Laet C, Kanis JA, Oden A, Johanson H, Johnell O, Delmas P (2005) Body mass index as a predictor of fracture risk: a metaanalysis. Osteoporos Int 16:1330-1338

23. Roy DK, O’Neill TW, Finn JD, Lunt M, Silman AJ, Felsenberg D (2003) Determinants of incident vertebral fracture in men and women: results from the European Prospective Osteoporosis Study (EPOS). Osteoporos Int 14:19-26

24. Samelson EJ, Hannan MT, Zhang Y, Genant HK, Felson DT (2006) Incidence and risk factors for vertebral fracture in women and men: 25-year follow-up results from the population-based Framingham study. J Bone Miner Res 21:1207-1214

25. van der Klift M, de Laet CE, McCloskey EV, Johnell O, Kanis JA, Hofman A (2004) Risk factors for incident vertebral fractures in men and women: the Rotterdam Study. J Bone Miner Res 19:1172-1180

26. Nevitt MC, Cummings SR, Stone KL, Palermo L, Black DM, Bauer DC (2005) Risk factors for a first-incident radiographic 
vertebral fracture in women $>$ or $=65$ years of age: the study of osteoporotic fractures. J Bone Miner Res 20:131-140

27. Vogt MT, Cauley JA, Kuller LH, Nevitt MC (1997) Bone mineral density and blood flow to the lower extremities: the study of osteoporotic fractures. J Bone Miner Res 12:283289

28. Pennisi P, Signorelli SS, Riccobene S, Celotta G, Di Pino L, La Malfa T (2004) Low bone density and abnormal bone turnover in patients with atherosclerosis of peripheral vessels. Osteoporos Int 15:389-395

29. Fahrleitner-Pammer A, Obernosterer A, Pilger E, Dobnig H, Dimai HP, Leb G (2005) Hypovitaminosis D, impaired bone turnover and low bone mass are common in patients with peripheral arterial disease. Osteoporos Int 16:319-324
30. Tanko LB, Bagger YZ, Christiansen C (2003) Low bone mineral density in the hip as a marker of advanced atherosclerosis in elderly women. Calcif Tissue Int 73:15-20

31. Schulz E, Arfai K, Liu X, Sayre J, Gilsanz V (2004) Aortic calcification and the risk of osteoporosis and fractures. J Clin Endocrinol Metab 89:4246-4253

32. Wong SY, Kwok T, Woo J, Lynn H, Griffith JF, Leung J (2005) Bone mineral density and the risk of peripheral arterial disease in men and women: results from Mr. and Ms Os, Hong Kong. Osteoporos Int 16:1933-1938

33. Crawford ST, Olsen RV, Pilgram TK, Duncan JR (2003) Validation of an angiographic method for estimating resting blood flow to distal tissue beds in the lower extremities. J Vasc Interv Radiol 14:555-565 